Article

\title{
Impact of neck and/or shoulder pain on headache
}

\author{
Charly Gaul ${ }^{1}$,Martin C. Michel 2,*, Anette Lampert ${ }^{3}$, Manuel Plomer ${ }^{3}$, Thomas Weiser ${ }^{3}$ and \\ Stefanie Förderreuther ${ }^{4}$ \\ 1 Migraine and Headache Clinic, Königstein im Taunus, Germany; c.gaul@migraene-klinik.de \\ 2 Dept. of Pharmacology, Johannes Gutenberg University, Mainz, Germany; marmiche@uni-mainz.de \\ ${ }^{3}$ Medical, Consumer Healthcare, Sanofi-Aventis Deutschland GmbH, Frankfurt am Main, Germany; \\ anette.lampert@sanofi.com (AL), manuel.plomer@sanofi.com (MP), Thomas.weiser@sanofi.com (TW) \\ ${ }^{4}$ Dept. of Neurology, Ludwig Maximilian University, Munich, Germany; \\ Steffi.foerderreuther@med.uni-muenchen.de \\ * Correspondence: marmiche@uni-mainz.de; Tel.: +49-6131-179346
}

\begin{abstract}
As neck and/or shoulder pain (NSP) frequently occur together with tension-type headache (TTH) and migraine, we explored how concomitant NSP affects perceived treatment responses to an analgesic. An anonymous survey was performed among 895 TTH and migraine sufferers who used the analgesic $400 \mathrm{mg}$ ibuprofen/100 mg caffeine. NSP was relatively abundant among patients (42.4\% for TTH; 39.2\% for migraine), and associated with $>1$ additional day with headache per month. Reported pain reduction was independent from NSP for TTH and migraine. More patients became pain-free at $2 \mathrm{~h}$ in migraine with NSP (42.9\%) compared to migraine without NSP (32.2\%), which is different from TTH with NSP (60.6\%) and TTH without NSP (71.4\%). For both, migraine and TTH, a recurrence of headache on the same day was more prevalent in those with concomitant NSP leading to a greater likelihood of taking a second dose of the analgesic. NSP frequently occurs together with TTH and migraine patients. In migraine, NSP seems to be associated with a better treatment response at $2 \mathrm{~h}$. The more frequent recurrence of pain in those with concomitant NSP indicates that NSP makes both headache types worse. Further studies are needed to substantiate these effects.
\end{abstract}

Keywords: tension-type headache; migraine; neck and shoulder pain; ibuprofen; caffeine

\section{Introduction}

Headache occurs frequently in the general population; for instance, migraine is ranked as \#2 in the top-15 in the WHO list of "Burden of Neurological disease [1]. Tension-type headache (TTH) and migraine are the two most common types of headache $[2,3]$. Neck and/or shoulder pain (NSP) is also very prevalent in the general population and even more frequent in subjects with concomitant TTH and/or migraine [4]. Increased pericranial tenderness is the most significant abnormal finding in TTH and very probably is of pathophysiological importance [5]. Conversely, NSP is a frequent comorbidity in TTH and migraine patients [5]. The comorbidity of migraine and NSP is so prevalent that it is debated whether NSP is a premonitory symptom or part of the migraine attack [6, 7]. The trigeminal-cervical complex could be a pathophysiological explanation of this association. A possible argument against a specific association between NSP and migraine is that a similarly tight association occurs between NSP and TTH [4].

The frequent comorbidity between TTH and migraine on the one and NSP on the other hand has several consequences. Firstly, comorbidity was reported to be associated with a lower level of 
physical activity and psychological well-being, a higher level of perceived stress and poorer self-rated health [8]. Second, those with this comorbidity were reported to have greater prevalence and severity of cutaneous allodynia [9]. Third, migraine may incorrectly be self-diagnosed as NSP [10] and the presence of NSP may delay initial migraine treatment beyond 30 minutes of headache onset and initiation of treatment at a greater headache pain intensity [11].

Analgesics are the most common form of acute headache treatment and triptans are used additionally in the subgroup suffering from migraine. Epidemiological data in Germany demonstrate that 3 out of 4 patients treat headache with over-the-counter (OTC) medications and that 3 out of 5 migraine patients do not regularly consult a physician regarding their headache [12]. Given the high prevalence of self-treatment of headache, the headache and migraine societies of German-speaking countries and the German Society of Neurology developed a guideline for the self-medication of common headache forms such as TTH and migraine [13].

To the best of our knowledge it is unknown whether and how concomitant NSP affects treatment responses to analgesics. Therefore, a pharmacy-based consumer survey was designed to explore the effect of concomitant NSP on the self-reported treatment response to an analgesic in TTH and migraine patients including onset of perceived pain relief. As example of this, we studied subjects performing self-treatment with an OTC fixed-dose combination of ibuprofen and caffeine $(\mathrm{I}+\mathrm{C})$. Our secondary objective was to gather further insight on the association between TTH and migraine on the one and NSP on the other hand at baseline.

\section{Experimental Section}

The present survey was performed in 126 community pharmacies in Germany and involved 1124 users of OTC for headache treatment including 192 healthcare professionals (HCP; pharmacists and pharmacy technicians). As data in pharmacy customers and HCPs were similar, they were pooled for all subsequent analyses. Subjects were offered to participate in the survey if they had purchased an OTC, branded fixed-dose combination of $400 \mathrm{mg}$ ibuprofen plus $100 \mathrm{mg}$ caffeine (Thomapyrin ${ }^{\circledR}$ Tension Duo). While all participants were included in the safety analysis, subjects were included in the analysis of baseline symptoms and effectiveness if they reported to use $\mathrm{I}+\mathrm{C}$ for the treatment of self-reported TTH or migraine (and excluded in case of other pain conditions or major data missing), leaving a total of 895 subjects to be analyzed (Figure 1). In the following we report the data for all TTH and migraine sufferers and within each group those with and without concomitant NSP. The survey did not require ethical committee approval because of being anonymous and non-interventional. Returning the anonymous survey was considered to represent consent to participate.

Parameters captured anonymously (paper-pencil) from users of $\mathrm{I}+\mathrm{C}$ included demographic variables, type of occupation (largely sitting vs. not), prevalence and amount of headache days in past month, type and intensity of pain on a numerical pain rating scale (NPRS; from $0=$ no pain to $10=$ worst pain) to be treated with $\mathrm{I}+\mathrm{C}$, perceived triggers of pain, subjective and objective measures of time to onset of pain relief after ingestion of first dose, pain intensity after $2 \mathrm{~h}$ (NPRS), \% of subjects being pain-free after $2 \mathrm{~h}$ (defined as " 0 " or " 1 " on NPRS) and global estimate of effectiveness and tolerability. AE reporting included perceived effectiveness or tolerability rated as not so good or poor. Handwritten personal notes on questionnaires were also checked. All disease assessments 
including classification whether the patient was suffering from TTH or migraine and assumptions on triggering factors were self-reported and did not involve a healthcare professional.

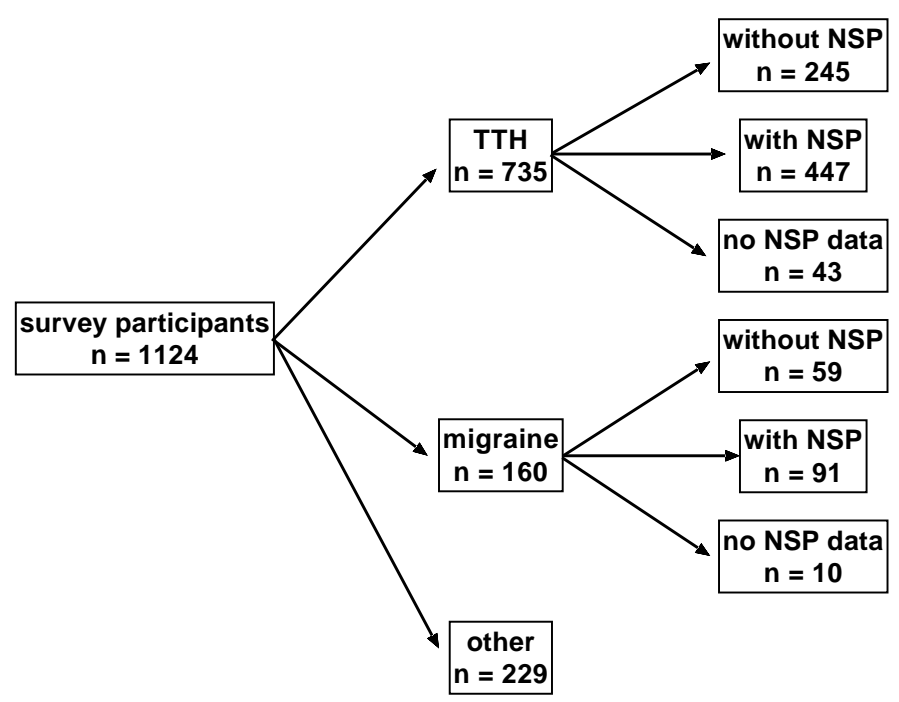

Figure 1: Disposition of participants. Note that the "other" group included 44 subjects reporting a combination of TTH and migraine, 44 reporting TTH or migraine in combination with additional pain types other than NSP, and 34 with major data missing.

Based on the exploratory nature of the survey, only descriptive and no hypothesis-testing statistics were applied. Data are reported as means $\pm \mathrm{SD}$ and/or as medians with interquartile ranges (IQR) for continuous and as percentage for categorical variables. In line with the exploratory character of the study, no hypothesis-testing statistical analysis was performed. The reporting in this manuscript adheres to the SRQR guidelines for qualitative research [14].

\section{Results}

Demographic data of the participants are shown in Table 1. More users reported TTH than those reporting migraine (735 vs. 160; Table 1). Migraine sufferers were more likely to be female ( 80.0 vs. $65.8 \%$ ), were slightly younger, and slightly more often reporting a largely sitting occupation. They reported a greater basal pain intensity than TTH patients (7.3 \pm 1.5 vs. $5.9 \pm 1.7$ on a NPRS), slightly more days with headache per months and almost twice as many days per month with impaired daily life (Table 1). Gender distribution, age and pain intensity within the TTH and migraine groups were comparable in the absence and presence of NSP. However, the presence of NSP was associated with more days per month with headache and with an impaired daily life (Table $1)$. 
Table 1: Demographics and baseline data according to type of headache in participants with and without concomitant NSP. Complaint data refer to last 30 days prior to purchase of medication. Data are means \pm SD for continuous variables (medians shown additionally in parentheses for some variables) and percentages for categorical variables. 43 subjects with TTH and 10 with migraine did not report on the presence or absence of NSP.

\begin{tabular}{|c|c|c|c|c|c|c|}
\hline \multicolumn{7}{|c|}{$\begin{array}{l}\text { Table 1: Demographics and baseline data according to type of headache in participants with and without concomitant NSP. Comp } \\
30 \text { days prior to purchase of medication. Data are means } \pm \text { SD for continuous variables (medians shown additionally in parenthes } \\
\text { and percentages for categorical variables. } 43 \text { subjects with TTH and } 10 \text { with migraine did not report on the presence or ab }\end{array}$} \\
\hline & \multicolumn{3}{|c|}{ TTH } & \multicolumn{3}{|c|}{ Migraine } \\
\hline & All & $\begin{array}{c}\text { Without } \\
\text { NSP }\end{array}$ & With NSP & All & $\begin{array}{c}\text { Without } \\
\text { NSP }\end{array}$ & With NSP \\
\hline $\mathrm{N}$ & 735 & 245 & 447 & 160 & 59 & 91 \\
\hline Age, years & $41.9 \pm 14.2$ & $40.7 \pm 14.6$ & $42.4 \pm 14.1$ & $40.2 \pm 12.9$ & $42.7 \pm 13.4$ & $39.2 \pm 12.3$ \\
\hline Gender, $\%$ female & 65.8 & 64.2 & 67.6 & 80.0 & 81.4 & 79.1 \\
\hline Mainly sitting activity, $\%$ & 35.5 & 29.6 & 38.3 & 39.6 & 27.6 & 47.3 \\
\hline Days with headache per month & $5.0 \pm 4.5(4)$ & $4.1 \pm 3.8(3)$ & $5.6 \pm 4.7(4)$ & $6.0 \pm 3.8(5)$ & $5.2 \pm 3.1(5)$ & $6.5 \pm 4.0(6)$ \\
\hline Days with impaired daily life per month & $1.3 \pm 2.9(0)$ & $0.7 \pm 1.7(0)$ & $1.6 \pm 3.3(0)$ & $2.4 \pm 2.7(1)$ & $1.7 \pm 2.3(1)$ & $2.3 \pm 3.0(1)$ \\
\hline Pain intensity, 0-10 NPRS & $5.9 \pm 1.7$ & $5.5 \pm 1.7$ & $6.0 \pm 1.8$ & $7.3 \pm 1.5$ & $7.2 \pm 1.6$ & $7.4 \pm 1.3$ \\
\hline
\end{tabular}


Subjects with NSP reported a greater prevalence of a largely sitting occupation and, accordingly considerably more often reported poor posture as perceived trigger, whereas stress and weather sensitivity were reported less often (Figure 2). Nutrition (for instance "not drinking enough") was perceived less often as a trigger with concomitant NSP in TTH, but more often in migraine patients; similarly, common cold and other diseases were perceived less often as trigger in TTH subjects with concomitant NSP, whereas no major differences were found in the migraine group.
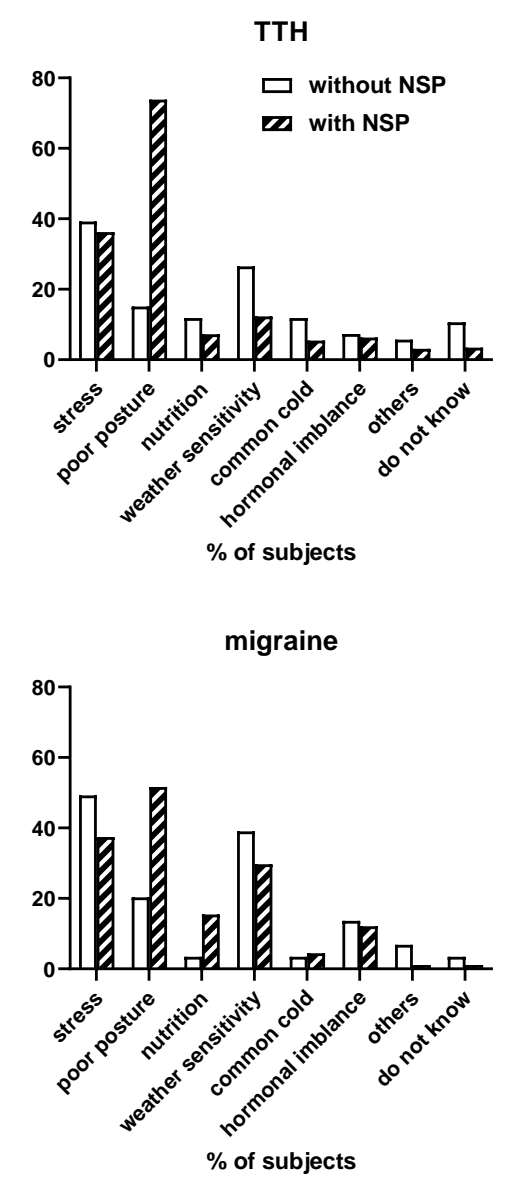

Figure 2: Perceived triggers in subjects with TTH (upper panel) and migraine (lower panel) with and without concomitant NSP. Data are shown as percentages; they do not add up to 100 because multiple answers were possible.

Two hours after intake of the first dose of $\mathrm{I}+\mathrm{C}$, pain reduction was similar for participants with TTH or migraine, irrespective of concomitant NSP and regardless whether assessed as difference of points on the NPRS or as percentage of participants with a reduction of pain intensity by at least $50 \%$ (Figure 3). While migraine patients reported becoming pain-free less often than TTH patients, the effect of concomitant NSP was inconsistent, i.e. associated with slightly fewer pain-free subjects in the TTH and slightly more pain-free subjects in the migraine group (Figure 3 ). The presence of concomitant NSP had little effect on the time of the day when the first dose of $\mathrm{I}+\mathrm{C}$ was administered in TTH patients. However, concomitant NSP was associated with a greater percentage of sufferers administering the first dose of $\mathrm{I}+\mathrm{C}$ in the morning in migraine patients and with a smaller percentage taking it in the afternoon (data not shown).

Time to onset of pain relief was similarly fast in subjects with TTH with or without NSP and in subjects with migraine with NSP with about $80 \%$ reporting onset within 30 min or less (Figure 4). Onset of pain relief was somewhat slower in subjects with migraine without NSP, but still about $66 \%$ of patients reported onset within 30 min or less. Accordingly, subjective assessment for time to onset 
was rated as very fast or fast by $79.2 \%$ of those with TTH without NSP, $83.9 \%$ with TTH with NSP and $80.2 \%$ with migraine with NSP, whereas it was $55.9 \%$ in those with migraine without NSP.11
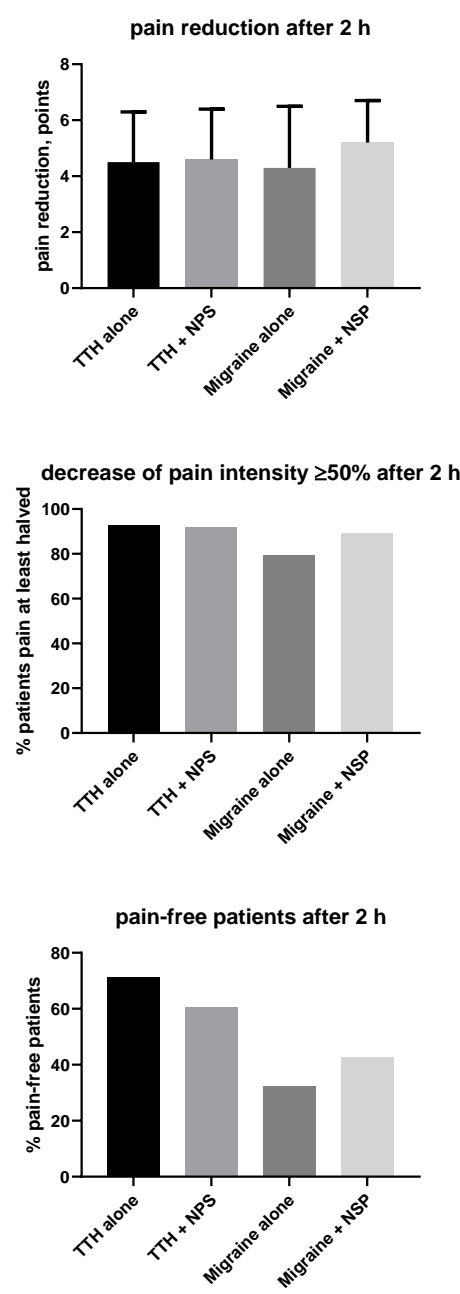

Figure 3: Effect of first dose of medication in participants with and without concomitant NSP. Data are shown as reduction in number of points \pm SD on a NPRS (upper panel), \% of patients reporting a symptom reduction by at least $50 \%$ (middle panel) and \% of patients reporting to be pain-free (lower panel; all relative to pain rating before administration of medication). All responses related to time point $2 \mathrm{~h}$ after intake.

The pain did not recur on the day of administration of I+C in $70.1 \%$ and $50.0 \%$ of TTH patients without and with NSP, respectively, whereas it did not return in $31.0 \%$ and $23.1 \%$ of migraine patients without and with NSP, respectively. If it returned, this was after more than $6 \mathrm{~h}$ in most patients, but in 55\% of patients with migraine without NSP it returned in less than $6 \mathrm{~h}$ (Table 2). Accordingly, use of more than 1 tablet of $\mathrm{I}+\mathrm{C}$ on the day of first administration was more frequent in subjects with migraine, and within each group more frequent with concomitant NSP (TTH without NSP 32.1\%, TTH with NSP 51.2\%, migraine without NSP 53.4\%, migraine with NSP 67.0\%). 

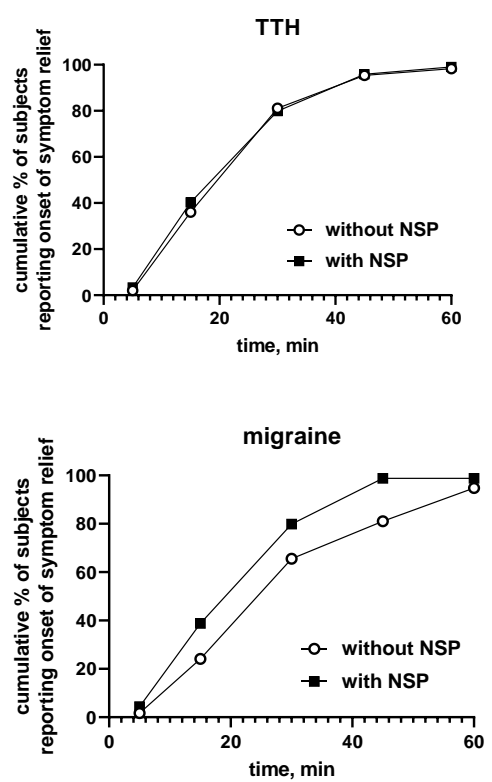

Figure 4: Time to onset of symptom relief in subjects with TTH (upper panel) and migraine (lower panel) with or without concomitant NSP. Data are shown as cumulative percentage of patients reporting onset of relief.

Table 2: Pain recurrence within the same pain episode. Data are shown as \% of subjects and absolute number in parentheses.

\begin{tabular}{|c|c|c|c|c|}
\hline & $\begin{array}{c}\text { TTH } \\
\text { with NSP } \\
\mathbf{( 4 4 7 )}\end{array}$ & $\begin{array}{c}\text { TTH } \\
\text { without NSP } \\
\mathbf{( 2 4 5 )}\end{array}$ & $\begin{array}{c}\text { Migraine } \\
\text { with NSP } \\
\mathbf{( 9 1 )}\end{array}$ & $\begin{array}{c}\text { Migraine } \\
\text { without NSP } \\
\mathbf{( 5 9 )}\end{array}$ \\
\hline Pain... & & & & \\
\hline did not recur & $50.0(222)$ & $70.1(169)$ & $23.1(21)$ & $31.0(18)$ \\
\hline recur after $>10 \mathrm{~h}$ & $19.4(86)$ & $9.1(22)$ & $30.8(28)$ & $20.7(12)$ \\
\hline recur after 7-10 h & $15.1(67)$ & $10.8(26)$ & $13.2(12)$ & $10.3(6)$ \\
\hline recur after 4-6 h & $13.3(59)$ & $7.1(17)$ & $24.2(22)$ & $17.2(10)$ \\
\hline recur after 1-3 h & $1.8(8)$ & $2.5(6)$ & $7.7(7)$ & $13.8(8)$ \\
\hline recur within 1 h & $0.5(2)$ & $0.4(1)$ & $1.1(1)$ & $6.9(4)$ \\
\hline Missing values & $(3)$ & $(4)$ & - & $(1)$ \\
\hline
\end{tabular}

Global tolerability was rated as very good (42.6\%), good $(54.1 \%)$, not so good $(3.2 \%)$ or poor $(0.1 \%)$ by the 1124 survey participants, i.e. including those with uses other than TTH or migraine. The percentage of subjects rating tolerability as very good or good was similar in TTH and migraine with and without concomitant NSP. Based on the definition of AE (including global effectiveness or tolerability rating as not so good or poor; see Methods), potential AEs were reported in 99 (8.8\%) out of 1124 participants. Among these were 63 reports with perceived effectiveness rated not so good (5.6\%), and 15 with poor (1.3\%); 35 with perceived tolerability rated not so good (3.1\%), and 2 with bad (0.2\%). Specific AEs based on handwritten notes include 1 case each of trembling, heart racing, stomach pain, dizziness and neck pain worsened. One participant reported in the handwritten notes to have taken $\mathrm{I}+\mathrm{C}$ for the indication fever, which is an off-label use and for this reason was counted as $\mathrm{AE}$.

\section{Discussion}

Our data confirm the frequent comorbidity between headache (TTH or migraine) and NSP. They show that $\mathrm{I}+\mathrm{C}$ is effective in subjects with TTH and concomitant NSP does not reduce its 
effectiveness. This also applies to migraine sufferers but this group reports a more frequent recurrence of pain within the same episode.

\subsection{Critique of methods}

The classification of patients as suffering from TTH or migraine was not based on a medical diagnosis but on self-assessment by the patients. However, we have previously found that a high percentage of patients can adequately self-classify their type of headache in line with the TTH definition of the International Classification of Headache Disorders [15].

The exploratory character of our study implies that no hypothesis-testing statistical analysis was applied to the data [16]. In line with the frequent use of self-treatment among TTH and migraine sufferers [12], we had chosen to perform a pharmacy-based consumer survey. This tool has proven useful in exploratory studies in various indications with frequent self-medication, for instance in the use of ambroxol in acute cough [17] or fixed-dose combination of ibuprofen and pseudoephedrine in upper respiratory tract infections associated to common cold [18]. While this tool is suitable for comparisons between groups, in contrast to randomized, controlled trials is not suitable to prove efficacy. As our study protocol did not specify a certain fraction of migraine patients among participants, we ultimately recruited a limited number of these, which makes conclusions on this group less robust than those with TTH. Moreover, data on physical activity, exercises or other instruments to overcome a more sitting activity have not been collected as part of this survey. These limitations notwithstanding, the differential baseline data (including gender and baseline pain) and medication use in TTH vs. migraine patients is in line with general observations in the headache field [5], indicating that the sample captured in our survey is representative of the overall $\mathrm{TTH} /$ migraine population.

\subsection{Findings at baseline}

A previous study reported that concomitant NSP was associated with a lower level of physical activity [8]. Similarly, our data show that subjects with concomitant NSP were more likely to have a mainly sitting activity ( $38.4 \%$ vs. $29.6 \%$ for TTH and $47.3 \%$ vs. $27.6 \%$ for migraine). Previous data also suggested that those with concomitant NSP had a lower level of psychological well-being, a higher level of perceived stress and poorer self-rated health [8]. In line with these findings, we observed that those with concomitant NSP had a slightly greater pain intensity and approximately one more day per month with headache and 0.6-0.9 more days per months with an impaired daily life (Table 2). This supports the observation that disability due to neck pain is more frequent in those with chronic as compared to episodic migraine and that conversely those with chronic migraine were at greater risk to have NSP-related disability [19].

Subjects with NSP reported partly different roles for some perceived triggers of their headache episodes. Most notably among those differences and in line with a greater prevalence of mainly sitting activities was a difference in self-reported poor posture $(73.8 \%$ vs. $15.1 \%$ in TTH, $51.6 \%$ vs. $20.3 \%$ in migraine). This is in line with a case-control study describing decreased muscle strength in the neck extensor muscles as a potential root-course of TTH [20]. Expectedly, subjects with migraine more often reported hormonal imbalance than those with TTH. While the perceived role of other triggers including nutrition (such as "not drinking enough"), common cold and other diseases also differed somewhat with concomitant NSP, these differences were much smaller than those of posture. Taken together these findings not only support the frequent comorbidity of headache and NSP but also strengthen the conclusion that concomitant NSP is associated with worse symptoms and impairment in both TTH and migraine sufferers [4]; however, they do not support the hypothesis that NSP should be considered as a premonitory symptom or part of migraine only $[6,7]$.

\subsection{Perceived treatment response}

As expected, migraine sufferers reported a greater pain intensity prior to intake of the first dose of medication than those with TTH. However, both groups experienced very similar reductions on pain intensity $2 \mathrm{~h}$ after intake of $\mathrm{I}+\mathrm{C}$, and concomitant presence of NSP did not change this. 
Similarly, the percentage of patients reporting an at least $50 \%$ reduction in pain intensity was comparable in those with TTH and migraine, and concomitant presence of NSP did not reduce those percentages. However, the percentage of pain free patients $2 \mathrm{~h}$ after intake of $\mathrm{I}+\mathrm{C}$ was lower in those with migraine than those with TTH; concomitant NSP did not affect this to a major degree. Taken together these data support the view that the fixed-dose combination of I+C is effective in TTH and migraine, whether concomitant NSP is present or not.

Among TTH patients, the perceived effectiveness of $\mathrm{I}+\mathrm{C}$ was mostly similar in those with and without concomitant NSP but some interesting exceptions were observed. Firstly, the percentage of TTH patients becoming pain-free was slightly smaller with than without NSP. Secondly, recurrence of pain and intake of a second $\mathrm{I}+\mathrm{C}$ tablet on the same day were more frequent with than without NSP. Taken together these data indicate that concomitant NSP is associated with symptoms that are more difficult to be controlled by medication in the TTH group.

Among migraine patients, concomitant NSP manifested in a different way than among TTH sufferers: Subject with NSP tended to take their medication earlier in the day and somewhat more often reported to become pain-free. However, they also reported more frequently that pain returned on the same day and that they ingested a second dose of $\mathrm{I}+\mathrm{C}$ on that day. Migraine sufferers with and without concomitant NSP considerably more frequently reported recurrence of pain within the same episode than those with TTH; a high recurrence rate within the same episode is common among migraine patients and also occurs with other types of medication such as triptans [21]. In contrast, migraine patients without NSP reported less often that pain halved and a somewhat slower onset of pain relief than the other three groups. Thus, the absence of NSP in migraine patients appears to be associated with symptoms that appear stronger and are not as responsive to drug treatment.

\section{Conclusions}

To the best of our knowledge, the present data describe for the first time the occurrence of NSP in a representative sample of headache sufferers treating their symptoms in self-medication and provide insight into the prevalence of this problem. Our data confirm that NSP is a medically important comorbidity among headache patients. While subjects with TTH or migraine with or without concomitant NSP reported a similar numerical degree of pain relief upon ingestion of $\mathrm{I}+\mathrm{C}$, our data also reveal differences between these groups - specifically how concomitant NSP affects individual parameters of the treatment response. More research is needed to verify these findings and to study whether this is also applicable to other headache treatments and whether concomitant NSP should play a role in the selection of the most appropriate treatment.

Author Contributions: Conceptualization, C.G., H.G. and A.L.; methodology, A.L. and M.C. M.; writing-original draft preparation, M.C.M.; writing-review and editing, C.G., H.G., A.L., M.P., T.W. and S.F..; supervision, M.P.; project administration, H.G. All authors have read and agreed to the published version of the manuscript.

Funding: The underlying study was funded by Sanofi-Aventis Deutschland GmbH.

Acknowledgments: In this section you can acknowledge any support given which is not covered by the author contribution or funding sections. This may include administrative and technical support, or donations in kind (e.g., materials used for experiments).

\section{Conflicts of Interest:}

CG received honoraria from Allergan Pharma, Cerbotec, Grünenthal, Hormosan Pharma, Lilly Germany, Novartis Pharma, Ratiopharm, Reckitt Benckiser, Sanofi Aventis and TEVA. MCM has received consultancy fees from Sanofi-Aventis. HG, AL, TW and MP are employees of Sanofi-Aventis. SF has received honoraria from Allergan Pharma, Astra Zeneca, Hormosan Pharma, Lilly Germany, Novartis Pharma, Sanofi-Aventis, and TEVA. The funder had no influence on the interpretation of the data but authors H.G., A.L., T.W. and M.P. contributed to the study as part of their employment by the funder 


\section{References}

1. Feigin, V.L., A.A. Abajobir, K.H. Abate, F. Abd-Allah, A.M. Abdulle, S.F. Abera, G.Y. Abyu, M.B. Ahmed, A.N. Aichour, I. Aichour, et al., Global, regional, and national burden of neurological disorders during 1990-2015: a systematic analysis for the Global Burden of Disease Study 2015. Lancet Neurol 2017. 16(11): p. 877-897. 10.1016/S1474-4422(17)30299-5

2. Straube, A., B. Aicher, S. Förderreuther, T. Eggert, J. Köppel, S. Möller, R. Schneider, and G. Haag, Period prevalence of self-reported headache in the general population in Germany from 1995-2005 and 2009: results from annual nationwide population-based cross-sectional surveys. J Headache Pain, 2013. 14(1): p. 11. 10.1186/1129-2377-14-11

3. Yoon, M.-S., Z. Katsarava, M. Obermann, G. Fritsche, M. Oezyurt, K. Kaesewinkel, A. Katsarova, I. Santowski, H. Diener, and S. Moebus, Prevalence of primary headaches in Germany: results of the German Headache Consortium Study. J Headache Pain, 2012. 13(3): p. 215-223. 10.1007/s10194-012-0425-x

4. Ashina, S., L. Bendtsen, A.C. Lyngberg, R.B. Lipton, N. Hajiyeva, and R. Jensen, Prevalence of neck pain in migraine and tension-type headache: A population study. Cephalalgia, 2015. 35(3): p. 211-219. 10.1177/0333102414535110

5. Headache Classification Committee of the International Headache Society, The International Classification of Headache Disorders, 3rd edition. Cephalalgia, 2018. 38(1): p. 1-211. 10.1177/0333102417738202

6. Lampl, C., M. Rudolph, C.I. Deligianni, and D.D. Mitsikostas, Neck pain in episodic migraine: premonitory symptom or part of the attack? J Headache Pain, 2015. 16(1): p. 80. 10.1186/s10194-015-0566-9

7. Özer, G. and N. Benlier, Neck pain: is it part of a migraine attack or a trigger before a migraine attack? Acta Neurol Belg, 2018. 10.1007/s13760-018-1030-9. 10.1007/s13760-018-1030-9

8. Krøll, L.S., C.S. Hammarlund, M.L. Westergaard, T. Nielsen, L.B. Sloth, R.H. Jensen, and G. Gard, Level of physical activity, well-being, stress and self-rated health in persons with migraine and co-existing tension-type headache and neck pain. J Headache Pain, 2017. 18(1): p. 46. 10.1186/s10194-017-0753-y

9. Bragatto, M.M., D. Bevilaqua-Grossi, M.T. Benatto, S.S. Lodovichi, C.F. Pinheiro, G.F. Carvalho, F. Dach, C. Fernández-de-las-Peñas, and L.L. Florencio, Is the presence of neck pain associated with more severe clinical presentation in patients with migraine? A cross-sectional study. Cephalalgia, 2019. 39(12): p. 1500-1508. 10.1177/0333102419854061

10. Viana, M., G. Sances, S. Terrazzino, T. Sprenger, G. Nappi, and C. Tassorelli, When cervical pain is actually migraine: An observational study in 207 patients. Cephalalgia, 2018. 38(2): p. 383-388. $10.1177 / 0333102416683917$

11. Calhoun, A.H., S. Ford, and A.P. Pruitt, Presence of neck pain may delay migraine treatment. Postgrad Med, 2011. 123(2): p. 163-168. 10.3810/pgm.2011.03.2274

12. Radtke, A. and H. Neuhauser, Prevalence and burden of headache and migraine in Germany. Headache: J Head Face Pain, 2009. 49(1): p. 79-89. 10.1111/j.1526-4610.2008.01263.x

13. Haag, G., H.-C. Diener, A. May, C. Meyer, H. Morck, A. Straube, P. Wessely, and S. Evers, Self-medication of migraine and tension-type headache: summary of the evidence-based recommendations of the Deutsche Migräne und Kopfschmerzgesellschaft (DMKG), the Deutsche Gesellschaft für Neurologie (DGN), the Österreichische Kopfschmerzgesellschaft (ÖKSG) and the Schweizerische Kopfwehgesellschaft (SKG). J Headache Pain, 2011. 12(2): p. 201-217. 10.1007/s10194-010-0266-4

14. O'Brien, B.C., I.B. Harris, T.J. Beckman, D.A. Reed, and D.A. Cook, Standards for Reporting Qualitative Research: A Synthesis of Recommendations. Acad Med, 2014. 89(9): p. 1245-1251. 10.1097/acm.0000000000000388

15. Gaul, C., H. Gräter, and T. Weiser, Results from a pharmacy-based patient survey on the use of a fixed combination analgesic containing acetylsalicylic acid, paracetamol and caffeine by self-diagnosing and self-treating patients. SpringerPlus, 2016. 5(1): p. 721. 10.1186/s40064-016-2369-0

16. Michel, M.C., T.J. Murphy, and H.J. Motulsky, New author guidelines for displaying data and reporting data analysis and statistical methods in experimental biology. J Pharmacol Exp Ther, 2020. 372(1): p. 136-147. 10.1124/jpet.119.264143

17. Kardos, P., K.-M. Beeh, U. Sent, T. Mueck, H. Gräter, and M.C. Michel, Characterization of differential patient profiles and therapeutic responses of pharmacy customers for four ambroxol formulations. BMC Pharmacol Toxicol, 2018. 19(1): p. 40. 10.1186/s40360-018-0229-y

18. Klimek, L., H. Schumacher, T. Schütt, H. Gräter, T. Mück, and M.C. Michel, Factors associated with efficacy of an ibuprofen/pseudoephedrine combination drug in pharmacy customers with common cold symptoms. Int J Clin Pract, 2017. 71(2): p. e12907. 10.1111/ijcp.12907 
19. Florencio, L.L., T.C. Chaves, G.F. Carvalho, M.C. Gonçalves, E.C.B. Casimiro, F. Dach, M.E. Bigal, and D. Bevilaqua-Grossi, Neck pain disability is related to the frequency of migraine attacks: a cross-sectional study. Headache: J Head Face Pain, 2014. 54(7): p. 1203-1210. 10.1111/head.12393

20. Madsen, B.K., K. Søgaard, L.L. Andersen, J.H. Skotte, and R.H. Jensen, Neck and shoulder muscle strength in patients with tension-type headache: A case-control study. Cephalalgia, 2016. 36(1): p. 29-36. 10.1177/0333102415576726

21. Géraud, G., C. Keywood, and J.M. Senard, Migraine headache recurrence: relationship to clinical, pharmacological, and pharmacokinetic properties of triptans. Headache: J Head Face Pain, 2003. 43(4): p. 376-388. 10.1046/j.1526-4610.2003.03073.x 